\section{An Adluress}

ON THE

\section{MODIFICATIONS AND IMPROVEMENTS IN OPERATIONS ON THE BILIARY PASSAGES.}

Delivered before the London:Polyclinic.

BY A. W. MAYO ROBSON, F.R.C.S., Consulting Surgeon to the General Infirmarv. Leeds; Vice President Royal College of Surgeons of England.

IT is sometimes useful in surgical as in other matters to pause and look backward, if this be done with a mind open to find fault with its own past acts. Such a process in my own case has led me to modify considerably and, I confidently believe, to improve my work in the branch of surgery about which I propose to speak.

After an experience of over a thousand operations in the upper abdomen, it is only natural that one should be more critical of one's own work and that of other surgeons, and only satisfied with something more nearly approaching perfection, though so far as the saving of life was concerned in my earlier operations, I was able to state in the preface to the first edition of Diseases of the Gall Bladder and Bile Ducts that:

I had never lost a single patient after any operation for gall stones in the absence of malignant disease. deep jaundice, or infective cholangitis, and that cholecystotomy for gall stones, even including the infective cholangitis and deeply-jaundiced cases, only showed a mortality of $x .7$ per cent.

I feel, therefore, that the proposition " as soon as gall stones give serious trouble their removal by operation is the most rational method of treatment" is one that can be safely supported, since it is only from the complications, which in many cases of cholelithiasis arise sooner or later, that any danger after operation need be apprehended.

I can see, however, that had I been operating on my early cases at the present time, several of those that required a second operation would have been cured at first. Moreover I believe that many of those in which the convalescence was prolonged would have been saved much time and inconvenience had the more thorough measures now adopted been followed out.

I will endeavour to point out in what manner my methods have changed, and also how my views on the subject of cholelithiasis and the operations for the disease have undergone some alteration.

When gall stones have once formed, no medicine, so far as I know, can dissolve them or produce permanent relief though much may be done by medical and general treatment for the relief of the catarrh so regularly associated with choleJithiasis, which may in fact bring on attacks not to be distinguished from true gall-stone seizures.

Medical treatment must therefore always be tried fully before surgical measures are resorted to, but I think we are now all agreed that if after a fair trial medical means fail, surgical treatment should be adopted before serious complications supervene and before the patient is reduced by jaundice, sappuration, or other untoward manifestation.

While cholecystotomy is generally recognized as the operation to be aimed at in the treatment of affections of the gall bladder and bile ducts, it is often impossible to say what operation will have to be done until the abdomen is opened and the exact state of affairs made out, for a contracted or dilated gall bladder, a suppurating or merely a distended viscus, concretions in the gall bladder or cystic or common ducts, the condition of the surrounding organs, the presence or absence of adhesions, and a host of other conditions, will all influence the subsequent action of the surgeon, who always begins the operation as an exploratory one, the subsequent steps being altered according to the circumstances mentioned.

No surgeon should attempt the removal of gall stones unless he is prepared for any of the various operations on the biliary passages, such äs choledochotomy or cholecystectomy, as it is almost impossible to say beforehand what may be re quired until the ducts have been explored by the fingers and the condition of the affected viscera ascertained; no operation should, as a rule, be concluded until it is clearly made out that the ducts, including the hepatic and common; are quite free from concretions, otherwise disappointment and dissatisfaction are certain to follow.

Since in the majority of cases, then, an operation for gall stones is in the first place simply exploratory, the actual operation on the gall bladder or bile ducts being only determined by the condition found when the abdomen is opened, it may be well for us first to consider a simple abdominal section in the gall-bladder region.

Preparation for Operation.

It may be convenient here, before considering the operation itself, to accede to a request to give some of the details which I follow out in my operative work generally, especially as they apply to the operations Fam about to describe.

First, as to the room in which the operation has to be performed. Any ordinary well-cleaned room having high windows so as to give good top light answers almost as well as an operating theatre. There is, of course, an advantage in having electric light, but, as I shall show, the operation I now perform on the bile passages is done close to the surface with few exceptions, not as formerly at a great depth, which necessitated a special electric lamp, and always a very good vertical or high oblique light.

The advantage of operating in a hospital or surgical home is that the surgeon, or his house-surgeon or assistant, is responsible not only for the operation but also for the after-attendance, a matter almost as important as the operation itself. Moreover, the surgeon can do his work better, and with greater confidence, where he is accustomed to operate, and where he is confident that all his directions before, at the time, and subsequently, will be carried out to the letter.

In this matter of where the operation should be done, the gurgeon who has to do the operation ought to make the selection, and the patient should abide by the decision with the full contidence that the operator will select the place where he can do his work to the best advantage of the patient.

I have seen several unsatisfactory cases in which patients insisted on having operations performed in their own homes which were utterly unsuitable for such a purpose, that would in all probability have done well had they been in a surgical home, where the complications causing the trouble could have have had immediate and skilful attention.

The surgeon ought also to be helped by his ordinary assistant in all serious operations such as I am about to describe, for a stranger, no matter how skilful, can never accommodate himself immediately to the needs of the operator, and it must be remembered that surgery is a fine art that can only be carried out with the greatest perfection under circumstances that are favourable to the artist.

The selection of the anaesthetist should also be with the operator for with a competent anaesthetist the surgeon can devote the whole of his mind to his own part of the work in hand, without having his attention diverted to make suggestions concerning the anaesthetic.

With regard to instruments, a gall-stione-Fcoop is the only special appliance I employ, and ali the instruments are boiled for half an hour before being used.

My sutures and ligatures are of formalin catgut prepared by the xylol process, which I described in the BRITISH MEdicaL JodRNAL, of September 27 th, 1902, p. 974. No. " 00 " size is used for ligatures, and No. "o" size for sutures; they are strong and reliably aseptic. Since the introduction of celluloid thread I have given up the use of silk for sutures, as the former is much stronger, ties with a very firm knot, and is easily sterilized by boiling. As it is not absorbable I only employ it for the outer or serous suture in stitching the incision in the duct in choledochotomy, and for this purpose the " 0 " green chromic catgut prepared by the xylol process answers equally well, as it does not become absorbed before the second or third week. As showing the disadvantage of non-absorbable sutures, a case came under my notice in which a silk suture used in a choledochotomy formed the nucleus of another gall stone, which fortunately passed without further operation.

As sponges I employ sterilized gauze swabs, but for keeping the viscera out of the way I use flat marine sponges, than which I find nothing to answer so well. The area of operation is surrounded by dry sterilized towels, sterilized by dry superheated steam for half an hour.

My assistant, dressers, and nurses all wear boiled rubber gloves, though personally I do not unless I have just had a septic case to operate on, as I find they impair my sense of touch and cause some delay, but I take especial care to scrub and wash several times before operating, and near the operating table I keep a bowl of .1 in 2,000 mercury biniodide solution in order to lave my hands from time to time during 
the course of the operation. The instruments are used out of $a \mathrm{I}$ in 40 absolute phenol solution and the sponges out of $I$ in 2,000 biniodide solution, but they are wrung quite dry before being used. Doubtless an ordinary saline solution would answer equally well for instruments and sponges but $I$ think not for the hands. The ligatures are used out of $a$ carbolic solution in spirit in which they are also stored.

The patient is prepared by having an aperient given so as to secure the bowels being moved the day before operation, and an enema is given the evening before if the operation is to take place early the next morning. If there is any feebleness of pulse, 5 minims of liq. strychninae are given subcutaneously on the afternoon and evening of the day before operation, and 10 minims as soon as the operation has begun. Should there be chronic jaundice or a tendency to haemorrhage, calcium chloride is given; for although there is a greater tendency to bleeding in chronic jaundice from pancreatic disease than when jaundice is due to gall-stone obstruction, I think there can be no doubt that in all cholaemic conditions the blood becomes so altered that the coagulability becomes seriously diminished, and that these factors demand serious attention before any operation is undertaken in cases of common-duct cholelithiasis. After reading Professor Wright's researches on the coagulability of the blood, ${ }^{1} \mathrm{my}$ mind was prepared to grasp the possibility of turning the experience gained on dogs to practical uses in the human subject, as I had lost two jaundiced patients, one in 1888 and one in 1890 . from persistent oozing of blood subsequent to operation. I therefore at once began to employ it in these cases, and with benefit, but it has been only within the last three years, since using calcium chloride in apparently heroic doses, that I have heen able to get at the real value of the drug, which is one I now always employ in jaundiced patients, both before operation in 30-gr. doses by the mouth, and afterwards in 6o-gr. doses by the rectum thrice daily for two or three days, or longer if neediul.

The skin of the patient over the operation area is prepared the day before by thoroughly washing with soft soap, or some soap, not necessarily antiseptic, that will give a good lather; if neediul, shaving is then done, and the whole area is gently rubbed with benzine. A dressing of lint, wet with $\mathrm{I}$ in 40 carbolic solution in water, is then applied, and over this oilskin or guttapercha tissue. The dressing is changed early the next morning, and the skin is thoroughly washed, so as to clear away all loose and sodden epithelium, after which another $I$ in 40 dressing is applied, to be removed on the operating table.

If the patient is feeble, a pint of normal saline solution with an ounce of brandy is given a short time before the operation.

As shock is intensified by exposure to cold, my patients are always enveloped in cotton wool, which is conveniently done by making a suit of gamgee tissue that can be readily run together by the nurses in an hour or two the day before operation. When operating in a theatre I employ a heated table, but elsewhere india-rubber hot-water bottles around the patient take its place.

It will be found that a firm sandbag. about 18 in. long by $6 \mathrm{in}$. wide and $3 \frac{1}{2}$ in. deep, covered with flannel, and placed on the operating table at the liver level, will push the spine forward, and with it the liver and bile ducts, so that the common and hepatic duets are brought several inches nearer to the surface. By opening out the costal angle and tending to make the intestines slip down from the liver, it acts like the Trendelenburg position in pelvic surgery.

Though this method, until I drew attention to it, does not seem to have been employed by others, I can, from ample experience, speak well of its great utility.

\section{Operation.}

Whereas I used formerly to make a vertical incision through the linea semilunaris, I now always make my incision over the middle of the right rectus in a line parallel with its fibres, which are then separated by the finger, the posterior sheath of the rectus and peritoneum being divided together. Where the gall bladder is distended and there is no jaundice, a emall incision of 2 or 3 in. only may be required; but when it is necessary to explore either the hepatic, common, or deeper part of the cystic duct, instead of prolonging the incision downwards as was formerly done, I now carry it upwards in the interval between the ensiform cartilage and the right costal margin as high as possible, thus exposing the upper surface of the liver very freely. It will be lound that by lifting the lower border of the liver in balk (if needful first drawing the organ downwards from under cover of the ribs), the whole of the gall bladder and the cystic and common ducts are brought quite close to the surface, and as the gall bladder is usually strong enough, my assistant can take hold of it with his fingers or forceps and by gentle traction can keep the parts well exposed, at the same time that,-by means of his left hand with a flat sponge under it, he retracts the left side of the wound and the viscera, which would otherwise fall over the ccmmon duct and impede the view.

It will now be observed that instead of the gall bladder and cystic duct taking a considerable angle with the common duct, an almost straight passage is found from the fundus of the gall bladder to the entrance of the bile duct into the duodenum, and if adhesions have been thoroughly separated the surgeon has immediately under his eye the whole length of the ducts with the head of the pancreas and duodenum. So complete is the exposure that if needful the peritoneum can be incised over the free border of the lesser omentum and the common duct separated from the hepatic artery and portal vein, but this is not necessary, except where a growth or glands have to be excised. The surgeon, whose hands are both free, can now deal with the gall bladder, cystic, common, or hepatic ducts quite easily ; for example, with his left fingerand thumb he can so manipulate the common or cystic duct as to render prominent any concretions, which can be directly cut down on, the edges of the cpening in the duct being caught. by pressure forceps. The assistant can now take hold of the forceps with his left hand, as they with the sponge will form a sufficient retractor, since the duct is so near the surface.

When the duct is incised there is usually a free flow of bile, which it must be remembered is probably infective, but by inserting a sponge in the kidney pouch and rapidly mopping up the bile as its flows, by means of sterilized gauze pads, any soiling of surrounding parts is avoided, and if thought necessary, the bulk of the infected bile can be drawn off by the aspirator either from the gall bladder or from the common duct above the obstruction before the incision into the bile passage is made.

After removing all obvious concretions, the fingers are passed behind the duodenum and along the course of the hepatic ducts to feel if other gall stones are hidden there, and when the common duct has been incised a gall-stone scoop, the only special instrument $I$ use, is passed into the primary division of the hepatic duct in the liver, and down to the duodenal orifice of the common bile duct, and if thought necessary to insure the opening into the duodenum being patent, a long probe is passed into the bowel.

The incision into the bile duct, if one has to be made, is now closed by an ordinary curved round needle held in the fingers without any needle holder, a continuous catgut suture being used for the margins of the duct proper, and a continuous fine green catgut or spun celluloid thread being employed to close the peritoneal edges of the duct.

Where the gall bladder is contracted and the pancreas is indurated and swollen from chronic pancreatitis, and likely to exert pressure for a time on the common duct, I insert a drainage tube directly into the duct, passing it upwards into the hepatic duct, and closing the opening around it by a pursestring suture, the tube being fixed into the opening by a catgut stitch which will hold for about a week; but where this is not done and the size of the gall bladder will permit it, I usually fix a drainage tube into the fundus of the gall bladder in the same way, as this drains away all infected bile and avoids pressure on the newly-sutured opening in the duct.

So easy is it to remove impacted stones after this method of exposure, that I now never spend a long time in manipulating stones impacted deeply even in the cystic duct, but at once incise the duct, remove the concretions, and close the opening without damaging the duct by much pressure and prolonged manipulation.

Although there is seldom any fear of leakage or of infection. yet, where the ducts have been incised and extensive ad. hesions separated, there is usually some tendency to pouring out of fluid in the first few hours. I therefore generally insert a gauze drain through a split draınage tube, bringin it out by the side of the gall-bladder drain. This is usually removed within twenty-four hours.

The wound is closed by continuous catgut sutures, first to peritoneum and deep rectus sheath, and next to the anterior rectus sheath. L3stly, the skin margins are brought together by means of a few interrupted silkworm gut sutures inserted quite an inch from the line of incision and brought out an inch beyond the incision on the other side so as to allow the 
edges to fall together without tension, thus securing union by first intention.

To those having little experience in these operations, the modifications I have employed may seem trivial, but to those who have experienced the difficulties of the ordinary operation of removing gall stones from a contracted gall bladder, or from the cystic or common ducts, I feel sure the method I have described, which enables the whole of the bile passages to be dealt with as a straight tube close to the surface, will be sufficiently appreciated.

In these operations I employ forcipressure for the immediate arrest of haemorrhage, but $I$ find it is more satisfactory, also, to ligature all the bleeding points, as in jaundiced cases the compressed and unligatured vessels are apt to bleed subsequently and to lead to complirations that are avoidable hy careful haemostasis. For the same reason, I prefer to divide and ligature firm visceral, especially hepatic, adhesions where that is practicable, rather than as formerly to separate them with the finger or tear them through.

If the liver be slightly torn in separating adhesions, the bleeding must be carefully arrested before the abdomen is closed. Sponge pressure is usually sufficient if the laceration be small, and this may be made more efficient by using at the same time a solution of adrenalin, but, if the laceration be extensive, deep catgut sutures, applied by means of a round intestinal needle, will usually accomplish the desired effect; or, this failing, gauze pressure, the plug being left in until it becomes loose, will be certain to answer.

Nothing can be simpler than an ordinary cholecystotomy with a distended gall bladder, or even with a gall bladder of ordinary size, where a small incision suffices to expose the sac, which is emptied by the aspirator. The collapsed sac is then brought through the wound, and surrounded by sterilized gauze; it is then incised through the point where the needle was inserted, and through the wound in the fundus the gall-stone scoop is inserted, and all gall stones are removed, a probe or the finger being employed to prove the ducts clear. A firm rubber tube, much firmer than the drainage tubes ordinarily sold, is then inserted from $\frac{1}{2}$ in. to $I$ in. into the gall bladder, the edges of the incision being drawn firmly around it by a catgut pursestring suture, which is tied and cut short, the tube being fixed in position by a catgut suture which transfixes the tube and the edges of the incision in the gall bladder. The edges of the incision in the gall bladder are then fixed to the aponeurosis by three or four catgut stitches, but never to the skin unless a permanent biliary fistula is intended. This tube is sufficiently long to pass into a bottle by the side of the patient, it drains all the bile away from the wound, and by the time the catgat has dissolved the wound will have healed by first intention, except where the tube was, and that part heals by granulation within the next week or two if the ducts are clear.

The following is an example out of many of a simple cholecystotomy:

$$
\text { CASE I.-Simple Cholecystotomy : Recovery. }
$$

Mrs. C., aged 4I, was admitte' to the Leeds Infirmary, March, 1899, on account of a tumour of the gall bladder, noticed a month, but following on gall-stone symptoms of several years duration. March 28th, chole cystotomy was pertormed, and after several ounces of straw-coloured fluid mixed with pus had been withdrawn through an aspirator needle, the gall bladder was opened, and fourteen facetted gall stones were removed from it and the cystic duct, the largest being the size of a small walnut, the smallest the size of a pea. Exploration within the duct by means of a long probe, and outside the duct by the finger in the abdomen, failed to discover any further obstruction. A drainage tube was inserted into the gall bladder, and surrounded by a pursestring suture; the edges of the opening were then fixed by sutures to the aponeurosis. Bile appeared on the dressing on the rollowing day, the drainage tube was removed on the sixth, and the sutures on the eighth; day. No bile was discharged alter the nlach, and tho watiod returned to her home on seventeenth day.

What has been called the "ideal" operation, in which the opening in the gall bladder is immediately closed and returned into the abdomen was suggested by I Langenbuch; bat as it does away with the beneficial effects of drainage, and is moreover attended with more risk than the ordinary operation I have described I never employ it.

Another modification suggested by. Bloch is that of performing cholecystotomy in two stages. The operation consists in incising the parietes until the peritoneum is reached, though not opened; the wound is then packed with gauze, and left for several days, when adhesions will have formed between the gall bladder and the parietal peritoneum. The gall bladder can then be safely opened. Or if the peritoneum be opened, the gall hladder is fired, but not opened until adhesions have formed.
As the method is only available for the simplest casesnamely, where the gall bladder is distended-as it does away with all chance of exploring the ducts by the fingers within the abdomen, and as it is frequently followed by fistula, it needs only to be mentioned to be condemned as clumsy and uncertain, and no safer than the ordinary operation of cholecystotomy.

To this opinion there may be one exception: the operation à deux temps may present advantages when cholecystotomy is being undertaken in the presence of chronic jaundice associated with distended gall bladder and a haemorrhagic tendency, as in such cases there is usually malignant disease either of the head of the pancreas or of the bile ducts ; and when the peritoneum is exposed, but not opened, pressure can be applied to arrest the oozing of blood, which cannot always be easily stopped by ligatures or forceps. Bloch. however, in his original papers, and again in the Revue de Chirurgie for 1895 , does not recommend the operation for this reason, but on account of the fear of septic contamination of the peritoneum, which ample experience proves to be groundless.

A simple operation like the example just given is quite the exception, the gall bladder being usually contracted and surrounded by adh+sions; moreover, in these cases the obstruction will usually be found in the cystic or common ducts. The advantage of the complete operation that I have described, in which the edge of the liver is lifted up and the bile passages brought well under view, will be experienced in this class of cases.

Management of Contracted Gall Bladder.

The next question will be, How is the contracted gall bladder to be dealt with? If it is too small to be brought to the parietes, but sufficiently large to admit a drainage tube, the method of fixing the tube by purse string suture just described will be quite safe, even if the opening in the gall bladder has to be left 2 in. or 3 in. from the suriace, for the omentum can be made to lie against the tube, and by the time that the catgut is dissolved a track of adhesions will have formed that will quite effectually prevent extravasation; but in order to make assurance doubly sure I frequently insert a small split drainage tube with a little gauze in it quite down to the gall bladder, and bring it out by the side of the first tube.

CASE II.-I8olation of a Drainage. Tract by Omentum and by Gauze. Mrs. N., aged 57 , seen with Dr. H. Well till eight months ago. Never had spasms or jtundice previously. Six attacks in eight mouths. The pairascapular region. Examination nezative but for tenderness over gals bladder.

Operation, December $x_{5}$ th, 1808. - Very adherent dilated stomach, with Operation, December 15 h, 1898.- Very adherent dilated stomach, with tion of adhesions and cholerystotomy performed, kut as the gall bladder could not be fixed to the parietes a tubo was fixed in it and isolated by iodoform gauze.

Result.-Good recovery; tube removed on ninth day; Fwell since Result.-

If the gall bladder be so contracted as to be incapable of admitting a tube it may either be closed by suture, the line of union being protected from hurtful leakage by a strip of gauze laid over it and brought to the surface through a rubber tube or the contracted and useless remains of the gall bladder may be removed by cholecystectomy.

Before I discovered the method for complete exposure of the whole biliary passages which I now emplny, I frequently left the shrivelled and useless gall bladder, after clearing out its contents; with the result that relapse sometimes occurred and cholecystectomy had subsequently to be faced, as in the following case :

CASE III.-Cholecystotomy: Recurrence of Symptoms: Cholecystectomy: Recovery.

Mr. A., aged 56, seen with Dr. C. and $\mathrm{Wr}$. A., of Nottingham, for loss of flesh, general ili-health, and frequently-recurring pains in the right hypochondrium, the illness being of several years' standing

Cholecystotomy, Beptember 4 th, 1898. - Contracted gall bladder with adhesions to surrounding parts, the result of gall-stone irritation. cholecystotomy performed, the gall bladder being isolated by a gauze drain. This was followed by rellef for some months, when the painfu attacks recurred, accompan by rigors and slighican

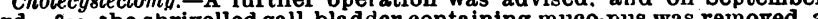
3rd, 1899, the shrivelled gall bladder containing muco-pus was removed, smail tube belng passed into and hxed in the cys creal in robust health, and said he had had no find when seen in

This and other similar cases of contracted gall bladder led me to adopt the operation of cholecystectomy in certain cases, where to leave the gall bladder means leaving a useless and diseased appendage lined with mucous membrane that is certain to secrete mucus, apt to be retained, owing to the cystic duct contracting and becoming strictured as the 
result of long-continued irritation and ulceration. This mucus, retained under tension, becomes infected, and a state of affairs much resembling chronic appendicitis is apt to continue, until either the gall bladder undergoes atrophy and absorption (a condition which may be termed cholecystitis obliterans, and which I have seen on several occasions) or the patient is worn out by repeated pain and chronic septicaemia, unless a further operation is undertaken to remove the offending organ.

Methods of Performing Cholecystectomy.

Cholecystectomy may be performed by three different methods, it being an important proviso that the common duct is cleared of concretions and freely patent.

It may be completely taken away and the cystic duct used as a pedicle and ligatured. When the gall bladder is very much contracted this is both easy and effectual, as the attachments to the liver are readily dissected off without tearing the liver substance. A method of treating the pedicle, otherwise the cystic duct, which I have found effectual but which to the best of my belief has not been adopted by others, is worth mentioning. The duct is seized with strong pressure forceps and crushed, thus making a groove in which the ligature, preferably of catgut, lies quite snugly. Any vessels that bleed are ligatured, and as a matter of precaution (for the parts being dealt with arenecessarily infected) a strip of iodoform gauze is left in contact with the end of the ligatured duct and brought to the surface through a split drainage tube. The following cases afford examples:

CASE -IV.-Removal of Gall Stone: Persistent Fistula: Cholecystectomy :

Mrs. S. G., aged 49, operated on in 1888 for gall stones (when sixty-six small ones were removed from a contracted and ulcerated gall bladde and cystic duct), was left with a mucous fistula which had to be kept open by a tube, as if it was allowed to heal pain and fever resulted. On May 14 th, 1890 , a further operation was performed. when the gall bladder was found shrivelled and adherent, and the cystic duct was strictured. The operation of cholecystectomy as just described was performed and a complete recovery resulted, the patient being well when heard of several years later.

CASE V.-Choledochotomy and Cholecystectomy : Recovery

Mrs. W., aged 57, seen with Dr. M., of Bolton, for repeated attacks of biliary colic associated with jaundice of three weeks' standing and rapid loss of flesh.

Operation June 8th, rgor, when a gall stone was found in the common duct and one in a calcareous gall bladder the shape and size of a hen's egg. Choledochotomy and cholecystectomy were performed and follow.

The second method is applicable to cases where the gall bladder is larger, but in which it is so seriously damaged as to be unsafe to leave it; or in cases where, owing to impaction of a concretion in the cystic duct, ulceration has occurred, and may be followed by stricture, which would both prevent the gall bladder performing its function as a reservoir for bile, and might lead to an accumulation of mucus and the formation of a tumour.

In this case the attachment to the liver is considerable, and, if the gall bladder be fully detached, laceration of the liver is apt to occur and troublesome bleeding, which can be saved by making a longitudinal incision through the fibrous coat on each side of the liver attachment, when the bulk of the gall bladder and all the mucous membrane can be removed, necessitating only iigature of branches of the cystic artery in the small portion of the fibrous coat left attached to the liver.

The cystic duct may then be ligatured, as described in the last operation, or plugged with a gauze drain, or drained by the insertion of a tube which is surrounded by a pursestring suture so as to prevent leakage of bile. The following cases afford examples :

CASE VI.-Cholecystectomy: Recovery.

Mr. M., aged 46, seen with Dr. R., of New York, and Dr. G., of Nice, for frequent seizures of intense pain, resembling biliary colic, with irregular fever and great loss of flesh and strength. At the operation, on May 2 nd, 895, I found an inflamed and contracted gall bladder, with cholangitis and extensive adhesions, doubtless due to gall stones that had passed. was excised in the way just described and the duct was plugged with gauze brought to the surface through a tube. Recovery was uninterrupted, and he was able to sail at the month end. Nine months later he was in perfect health.

CASE VII.-Gangrene of Gall Bladder : Partial Cholecystectomy : Recovery. Mr. M. A., aged 50 , seen with Dr. A., of York, January roth, rooo, for acute local peritonitis of a week's duration, starting in the region of the gall bladder, and ushered in by a rigor followed by fever and intense pain having only been noticed a month previously.

The operation was performed the same day, when gangrene of the fundus of the gall bladder was discovered, with intense local peritonitis, limited by acutely inflamed and darkened omentum, the patient being a very fat subject. Cholecystectomy performed, the portion of gall bladaer attached to the liver was left. as it was not gangrenous: a tube was inserted into the cystic duct. He made an uninterrupted recovery, and

Dr. W. Mayo of Rochester, U.S.A., suggested another method of partial cholecystectomy which he has found of service. He lays open the gall bladder and shells out the mucous membrane, if possible in one piece, plugging the cavity remaining with gauze. In some cases this is easy, but in others, where there has been much inflammatory disturbance, I have found it impracticable.

After cholecystectomy, in the case of a dilated cystic duct, where the ultimate patency of the common duct is question able, the open end of the duct may be connected to the bowel by choledoch-enterostomy, as in the following case :

CASE vII.-Mucous Fistula: Cholecystectomy: Recovery.

Mr. P. aged 55, seen at the Leeds Intirmary, July 24 th, 1895 . Operation undertaken for closing a mucous tistula, when the gall bladder was foun to be forming a tumour with walls $\frac{1}{2}$ to $\frac{3}{4}$ in. thick. Cholecystectomy wa performed, and the open end of the cystic duct connected to the smal bowel by means of a Murphy's button. A smooth recovery followed, and when heard of in 1896 he was completely cured and in good health.

If, as frequently happens, gall stones are so firmly fixed in the cystic duct that they cannot be pressed backward into the gall bladder, it is better not to use force but to incise the duct over the stone, and, after clearing the duct, to close it by a double row of sutures to mucous membrane and serous coat respectively, as in the following case :

CASE IX.-Empyema of Gall Bladder : Choledochotomy and Drainage Mrs. G., aged 47, seen with Dr. L., of Farsley. Repeated gall-stone seizures for two years; no jaundice ; no fever; distended gall bladder, ch is tender.

Operation, March 23 rd, 1899.-Empyema of gall bladder with many small stones in gall bladder; one large impacted stone, which could not be disdochotomy: drainage of gall bladder and gauze drain down to incision in duct. Good recovery. Well igor.

If the concretions are in the common duct either fixed or floating it is just as easy, with the duct well under the eye and near the surface, to incise it and remove the stones as it is to open the gall bladder. All such instruments as handled needles and Halsted's hammer are quite unnecessary, for the incision in the duct can as readily be closed by means of a curved round needle (sewing needle pattern) in the fingers as by any more complicated apparatus. But before closing the duct it is of the utmost importance to ascertain that there are no stones left either in the ampulla of Vater or in the hepatic duct; and although the fingers manipulating the outside of the ducts can give information as to any large stones, it would be easy to overlook small ones unless the scoop is passed freely upwards into the hepatic ducts and downwards behind the duodenum. or, if necessary, the opening in the duct can be made sufficiently large to admit the finger for exploration.

I usually pass a large probe down into the duodenum through the papilla to be sure that the passage is quite free. In one case, not being able to pass the probe beyond the papilla, I opened the duodenum and found a stricture of the common duct close to its termination, which $I$ divided after freely laying the papilla cpen over a director.

The following are examples of choledochotomy:

CASE X.-Choledochotomy.

A man, aged so years, was seen by me with Dr. W., of Ovenden. There had been gall-stone colic for two years and three months, with loss of flesh and strength. Jaundice and infective cholangitis were present. A stone was floating in the common dust, and there was a fistula between the gall bladder and the colon. On January 28 th, 1900, a gall stone was removed from the common duct by choledochotomy the fistula between recovery followed. The patient was quite well in September, rgor.

$$
\text { CASE XI.-Choledochotomy: }
$$

A man, aged 53 years, who was seen by me at the General Infirmary, had had colic for twelve years and jaundice for two years; the jaundice having been very deep for some time. On October roth. rgor, choledochotomy was performed in the manner previously described, and a stone was 1901.

$$
\text { CASE XII.-Choledochotomy. }
$$

A man, aged 58 years, was seen by me with Dr. $\dot{R}$., of Preston. He had had very deep jaundice for three years. Biliary cirrhosis and ascites as well as jaundice were present, and there was a stone in the common duct. On October 21st, r901, choledochotomy was performed, and the omentum was fixed to the anterior abdominal wall in order to cure the ascites. Anterior drainage was employed. In December, Igor. I had a letter to say that he was regaining strength and feeling well, and I am told that he has completely regained his health.

In quite a number of cases, after the common duct has been cleared, I have fonnd stones in the hepatic ducts, 
which I have removed by the scoop. The following are examples:

Case XIII.-Calculi in Bladder and Ducts: Choledochotomy : Recovery A policeman, aged 48 , a stout, unhealthy subject, with a history of former intemperance, had suffered from gall stones for twenty years, and from jaundice with infective oholangitis since May, Igor. On December 7 th, rgor, choledochotomy was performed in the infirmary, when 126 gall stones were removed from the gall bladder and 88 from the common and hepatic ducts. Recovery was retarded by bronchi
but the patient was discharged in five weeks, and has remained well:

CASE Xiv.-Calculi in Bladder and Ducts'; Cholédochotomy : Recovery.

On July r7th, r9o2, I operated on a lady, aged 70 , and removed a considerable number of gall stones from both arms of the hepatic duct after clearing the common duct of concretions through a choledochotomy opening. The patient is, I am informed, now quite well.

In some cases the common bile duct is found dilated to the size of the small intestine, and, if the gall bladder and cystic duct are small and so contracted as to be useless for drainage, a firm rubber tube is inserted into the incision in the duct and pushed a little way.up into the hepatic duct, the tube being surrounded by a pursestring suture and fixed in position by one or two catgut stitches, as in the following case:

$$
\text { CASE XV.-Choledochotomy : Drainage: Recovery. }
$$

$I$ saw the patient a man, aged $5 \mathrm{I}$ with Dr. H., of Upwell. There had been only slight colic, but for a year deep jaundice, with great wasting and infective cholangitis. Choledochotomy was performed on September 29th, Igor, five stones being removed from the common duct. As there was chronic pancreatitis, the dilated common duct was drained by means of a rubber tube fixed in by suture and surrounded by a pursestring suture. A lumbardrain was also inserted, but as events proved ist. 3 lb. in weight by November, Igor. He remained well when heard of in June, roor.

DUODENo-Choledochotomy.

If a stone is impacted in the duodenal end of the common duct it may sometimes be more easily reached through a vertical incision in the second part of the duodenum; when the concretions can be directly. cut down on through the posterior wall of the duodenum, or the papilla can be laid open over a director; it is then easy to pass the gall-stone scoop up the common duct in order to be certain that it is free from concretions. All that is now necessary is to close the anterior duodenal wound by a continuous catgut suture for the mucous membrane, and a continuous silk or celluloid thread for the serous margins. The following cases are given as examples :

A man, aged XSE XVI.-Dundeno-choledochotomy: Recovery. been colic and jaundice for six months with loss of weight and strength. Deep jaundice was present; there were gall stones in the common duct: in a good recovery, and the patient was well some mouths later.

CASE XVIr.-Duodeno-choledochotomy : Recovery.

woman, aged 49 , was seen by me at the Infirmary. For six years colic had persisted with varying jaundice. On March 22nd, 190r, duodenocholedochotomy was performed, and eight large, together with numerous small, gall stones: were removed from the common duct. good recovery ensued, the patient being well some months later.

With the better exposure of the common duct secured by the new operation, duodeno-choledochotomy will be seldom called for, as the gall-stone scoop can so freely be used through the incised duct.

\section{Cholmcystenterostomy.}

Cholecystenterostomy has been advocated by some surgeons for obstruction in the common duct by gall stones, the gall bladder being connected to the duodenum or colon. In my earlier practice I performed a number of these operations, but since I discovered the easy and effectual method of exposing the whole length of the bile ducts I have practicaily discarded this operation, for it leaves the cause untouched, and should the artificial opening close, the symptoms inevitably return, as in a case of chronic pancreatitis on which $I$ operated in November, 1899, where the communication was effected by a Murphy buttion, and though the patient was relieved for a time, the symptoms returned owing to the closure of the fistula:

The juaction of the viscera may be effected either by means of a small bone bobbin or by a Murphy button. As to the point of junction, the cases in which I have joined the gall bladder to the colon have done quite as well as those in which a duodenal junction was effected; and as the operation is very much easier I should certainly advocate and practise it in future, if called on to perform cholecystenterostomy simply as a means of giving relief.

The following is a description of a case of gall-bladder colon operation :

CAsE XvinI.-Cholecystotomy : Biliary Fistula: Cholecystenterostomy: On January gth, 1888, a married woman, aged 42, was admitted to the infirmarv suffering from acute local peritonitis, with a tumour in the region of the gall bladder.

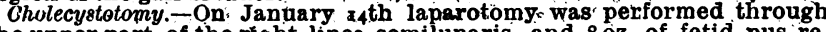
the.upper part of the right linea semilunaris, and .8 oz of fetid pus re moved from the gall bladder. Exploration of the ducts by the finger and a probe failed to discover any gajl stones. The gall bladder was stitched recovery, but with a biliary fistula. Although she had retained good recovery, but with a biliary fistula. Although she had retained good health during the fifteen months, when the fistula was open and discharging the whole of the bile, her condition was a very niserable one, was walking about, and her dressings and clothes beca me saturated.

Cholecystenterostomy was performed on March $2 \mathrm{nd}$, a 889 , by. reopening the abdomen through the old cicatrix in the right linea semilunaris. The viscera in the neighbourhood were found to be so matted together that it seemed to be impossible to fix the gall bladder to the duodenum, and as the hepatic flexure of the colon was eonveniently near, the gall bladder. was fixed to it by a double row of sutures 'round a decalcified bone' bobbin, a free communication being made between the two viscera. After a tardy convalescence she completely recovered, and was well in every respect ten years later.

The only cases in which I should now think it justifiable to perform cholecystenterostomy are those in which the obstruction is a permanent one-ior instance, in cancer of the head of the pancreas and growth or stricture of the common duct.

Marignant Disease.

Where gall stones are associated with cancer of the gall bladder, liver, or pylorus, a much more extensive operation may have to be done, as in the following cases:

CASE XIX.-Excision of Cancer of Liver and Gall Bladder; Recovery. A man, aged 46. Seven years gall-stone attacks. Infective cholangitis. Loss of 4 st. in weight. Jaundice. Operation, June 26 th, 1899 . Tumour of liver adjoining gall bladder excised by wedge shaped incision. Fundus of gall bladder also removed. Large number of gall stones removed, and cholecystenterostomy performed... Complete and perfect recovery. tion. Microscope showed disease removed to be cancer.

CASE XX.-Excision of Cancer from Liver, Gall Bladder, and Pylorus : Recovery.

A woman, aged 63. History of pain and jaundice. Great loss of flesh and strength. Tumour in gall-bladder region.

Operation, August roth, 3900 - Mass of growth discovered in liver, gall bladder, and pylorus. Cholecystectomy. pylorectemy, and partial hepatectomy performed. Good recovery. Patient well, and in good health now. Microscopic examination showed the disease to be cancer.

But these are exceptional cases, as there were no secondary manifestations of disease in the liver or elsewhere. Had there been such, I should have simply concluded the operation as an exploratory one.

\section{Intervisceral Fistula.}

In detaching adhesions it may be found that there is a fistula between the gall bladder and stomach, pylorus or bowel, and a careful search must always be made for this, lest an opening into one of the hollow viscera be left, which would probably mean extravasation and death. The following are. examples of intervisceral fistula.

CASE XXI.-Closure of Fistula between Gall'Bladder and Stomach : Recovery. Mrs. H. aged 6o, seen with Dr. C., of Doncaster. Aitarks of gall-ston pain for fifteen months, lately very frequent. and followed by slight jaundice.

Operation, July 7 th, 1898 . The stomach was found firm]y adherent to the gall bladder and on separating the adhesions a fistula between the gall bladder and stomach was found. The edges of the stomach fistula were pared, and the opening was closed with two rows of sutures Through the fistula in the gall bladder the gall stones were removed and drainage carried out. The patient made an excellent recovery and is now in good health.

CaSE XXII.-Closure of Fistula between Gall Bladder an' Colon : Recovery. Mr. G.; aged 50, seen with Dr. W. No history of spasms. First attack. of gall-stone colic in October, 1897 , followed by jaundice: severe seizures in December, $x 897$, with jaundice lasting two months and associated with ague-like seizures. Slight attacks for a year, and then one very severe in December, 1898 , and again in January, 1899 . During the whole period the icterus deepened after each attack, and occasionally rigors occurred ; lost over $2 \frac{1}{2}$ st. in weight; jaundiced but not deeply: Jiver not enlarged :
no tumour of gall bladder : tenderness above and to right of umbilicus : well-marked ailatation of stomach

Operation, January 28th, 1900. - Fistula between shrunken gall bladder and colon discovered, cystic duct shrunken; common duct dilated to size of small intestine, and containing large foating gall stone; calculus crushed, and fragments manipulated back through cystic duct; tube introduced into common duct through cystic duct; fistulous opening into colon closed. Uninterrupted recovery.

Garl Stonks and Pyrortc Stenosis.

If with the gall stones pyloric stenosis be fuund, then pyloroplasty or gastro-enterostomy will have to be performed, as in the following case:

CaSE XXIII_-Cholecystotomy, Choledochotomy, Puloroplasty. Miss T., aged 15 seen with Dr. W., of Harrogate History of gall-ston attacks, with slight jaundice for several years. History of gastric ulcera; tion with stomach symptoms for two years. Great loss of weight and but no tumour. 
Operation, August 5th, rgor.-Pyloric stenosis, with dilatation of stomach and gall stones, in gall bladder and common duct found; twenty gall stones removed from gall bladder by cholecystotomy, and two removed from common duct by cholednchotouy. Pylorus treated by pyloroplasty Smooth and rapid recovery. July, I902: Patient very well, and had gained over 2 st. in weight.

Cholelithiasis and Eniargenent of Pancreas.

In common-duct cholelithiasis, especially where there is a small floating gall stone, it is common to find the head of the pancreas enlarged and hard, the result of chronic pancreatitis, this will give rise to the suspicion of cancer of the head of the pancreas, and may lead to an unfavourable prognosis being given, but the following out of many cases that I have had shows that it is well to reserve our opinion in such cases, and to give the patient the hope of cure.

CASE XXIv. - Tnlargement of Head of Pancreas suggesting Malignant Disease: Cholecystenterostomy : Recovery.

On October 20 th, 18,9 , I was asked by Dr. S., of Sunderland, to see a lady, aged 5 , who had been suffering for three years from attacks resembling hose of gall stones, each attack being followed hy jaundice. past fourteen weeks the selzures had been more frequent and severe, and aundice had never quite cleared away before another attack came. She had lost flesh and strength considerably, and had vomited from time to time between the attacks. Her digestion was much impaired, and there was a want of appetite. She time of eacl seizure. The urine concained abundant Jithates and a slight trace of albumen, but no sugar. On examination the abdomen showed no manifest enlargement of the liver or gall bladder, but some tenderness over the gall bladder and at the epigastrium, where there was an indefinite sense of fullness.

Operation, October 23 rd.-After detaching numerous adhesions fifteen gall stones were removed from the cystic and common ducts, but as a large nodular mass was occupying the head of the pancreas and partly obstructing the common duct, it was deemed advisable to perform cholecystenterostomy, so as to make a permanent opening between the fundus of the gall bladder and the duodenum. The tumour gave the impression both to myself and to Dr. S., that it was malignant. Recovery was, howerer, uninterrupted, the button was passed on the tentil day, to put on flesh. She returned home, and has since been perfectly well in very respect. It is now over three years since the operation, and her health, I am informed, is perfect.

I have entirely given up cholelithotrity as a set operation, as although in my earlier cases it answered well in some cases, in several the fragments did not pass, and gave further trouble or led to relapse. Moreover, the very comp'ete exposure which the operation I have described gives renders all uncertain methods, such as crushing, quite unnecessary.

It would be easy from my own experience to add to the number of examples of each operation that I have described. Some hundreds of cases have been reported or referred to in the second edition of Diseases of the Gall Bladder and Bile Ducts.

\section{AFTER-TREATMENT.}

It only remains for me now to refer to the after-treatment which I previously said that I consider almost as important as the operation itself.

Expedition in operating is an important factor in lessening shock, especially in abdominal surgery, for it stands to reason that prolonged manipulation and exposure of the viscera, in patients so ill as the class of cases we are now considering must generally be, will be badly borne, for it is not only the work of the surgeon but the deep anaesthesia that adds to the shock, since for these operations to be expeditiously performed the muscles must be well relaxed. Choledochotomy should occupy from half an hour to an hour, and only in case of unusual complications a little longer.

After operation a pint of saline fluid with $1 \mathrm{oz}$. of brandy is given by enema, and 5 minims of liq. strychninae are given subcutaneously, this being repeated if called for. Subcutaneous injections of saline fluid or intravenous infusion are only rarely required.

Beyond teaspoonfuls of hot water or hot tea from time to time all feeding is by the rectum for the first twenty-four hours, though if there is no vomiting the teaspoonful of water s increased to a tablespoonful, or even two, every hour. After forty-eight hours, if there is no vomiting, milk and soda and barley-water can be freely given. A little plasmon dissolved in the tea or beef-tea or barley-water considerably adds to the nutritive value of the fluid. Light custard pudding is usually given on the fourth day, fish on the fifth, and chicken on the sixth, after which the diet becomes almost normal.

The bowels are not disturbed before the fifth or sixth day, and then only by enema, unless there is vomiting or distension, and in case of either of these complications a grain of calomel is administered, and followed by $2 \mathrm{oz}$. of Apenta water every two hours until it acts or until flatus passes freely, this being at times helped by the rectal tube or by a turnentine enema

Morphine is avoided after all my ab lominal operations, as it tends to paralyse the intestines and leads to an accumulation of flatus. I believe that abstention from the use of morphine is a great feature in the success of abdominal surgery, just as I feel sure that in the past it has killed many patients who would otherwise have done well.

If a sedative is needed $10 \mathrm{gr}$. of aspirin will be found useful, and this can be repeated in two hours if required. In case of vomiting being troublesome or epigastric distension persisting gastric lavage will be found useful, and when the stomach is emptied a dose of Apenta water may be left in it to incite peristalsis. Under these circumstances no food or fluid is allowed by the mouth, but plenty of fluid in the shape of normal saline is given by rectum.

As a rule recovery is uneventful, and for the most part after-treatment is negative. The stitches are removed on the eight day, and the tube usually comes away about the same time; the wound generally will have healed by first intention, and the spot where the tube was heals by granulation. The dressings are of the simplest-sterilized gauze and sterilized wool being employed as a rule, double cyanide gauze just boiled being sometimes used next to the wound.

The chief lessons I have to teach are that we should operate earlier before serious complications have ensued, and that when we do operate we should be thorough, expeditious, and careful.

1 BRITISH MEdical JoURNAL, December i8th, r8gr.

\section{THE OPERATION OF CHOLECYSTECTOMY, WITH}

\section{A RECORD OF CASES."}

By B. G. A. MOYNIHAN, M.S., F.R.C.S.,

Senior Assistant Surgeon. Leeds General Infirmary; Consulting surgeon to the Skipton Hospital and to the Mirfeld Memorial Hospital.

THE operation of cholecystectomy-removal of the gallibladder-first performed on July 15 th, 1882, by Langenbuch, is one which is comparatively rare in the surgery of the bile passages. It is called for in the following conditions :

r. In injuries of the gall bladder, rupture, stab, or bullet wounds.

2. In gangrene of the gall bladder.

3. In phlegmionous cholecystitis.

4. In chronic cholecystitis with dense thickening of the walls of the gall bladder and cystic duct. with or without stenosis of the cystic duct. and in chronic cholecystitis. when the gall bladder is shrivelled and puckered, and universally adherent. In such cases it is no longer a receptacle for the bile.

6. In distension of the gall bladder, hydrops or empyema, due to block. age of the cystic duct by calculus, stricture, growth, or external inflanmatory deposits; or in cases of mucous fistula following operations for these conditions.

7. In cases of fistula between the gall bladder or the cystic duct on the one hand, and the stomach, duodenum, or colon on the other.

8. In multiple ulcerations of the gall bladder or the cystic duct, when gail stones have eroded their way through the walls into the liver, the duodenum, or other protective adherent masses.

In primary carcinoma of the gall bladder.

In all these conditions the removal of the gall bladder is either imperative or desirable. Kehr, whose experience of the surgery of gall stones is unrivalled, has recommended, as a routine measure in the treatment of cholelithiasis, the removal of the gall bladder and the drainage of the hepatic duct. This advice is based upon the belief that so radical a measure will lessen the likelihood of recurrence of the stones, -a belief which is probably chimerical, for we know beyond. dispute that gall stones are not iufrequently formed in the ducts as well as in the gall bladder. The recurrence of gall stones in his patients lessens with the increasing experience of the operator, and is best guarded against by a complete exploration of all the ducts in all operations. If this be done, the surgeon will occasionally find $a$ stone or stones in the hepatic or in the common duct, when no symptoms have been elicited by their presence there. The complete and minute examination of all the ducts is quite easily performed if the ordinary vertical incision be prolonged upwards along the costal margin and the liver rotated by gentle traction in a forward and upward direction upon the gall bladder.

In cases of recent acute or subacute cholecystitis, due to blockage of the cystic duct by a large stone, removal of the gall bladder is certainly not necessary. I have had several such cases under my. care, and in all drainage of the gall bladder for seven to ten days has been ample to permit of a subsidence of the acute inflammation. The gall bladder should never be removed unless the surgeon is convinced that the common duct is patent.

* A paper read before the Yorkshire Branch of the British Medical Assceiation. 